\title{
Cellular Physiology

\section{Hypertonicity-induced Mitochondrial Membrane Permeability in Renal Medullary Interstitial Cells: Protective Role of Osmolytes}

\author{
Li Zhang ${ }^{2}$, Dong Chen ${ }^{1}$, Zhonghai Chen ${ }^{1}$ and Gilbert W. Moeckel ${ }^{1,2}$
}

${ }^{1}$ Renal Pathology and Electron Microscopy Laboratory, Department of Pathology, Yale University School of Medicine, New Haven, ${ }^{2}$ Department of Pathology, Vanderbilt University Medical Center, Nashville

\section{Key Words}

Hypertonicity $\cdot$ Apoptosis $・$ Mitochondria $\cdot \mathrm{Bcl} 2 \cdot \mathrm{Bax}$

- Osmolytes

\begin{abstract}
Background: Hyperosmotic stress causes cell death through activation of apoptotic pathways if the protective osmolyte response is impaired. In this study we attempt to elucidate the molecular mechanisms of hypertonicity-induced apoptosis and the effect of major organic osmolytes upon those. Methods: Hypertonicity-induced changes in Bcl2-family protein abundance and the presence of cytochrome $c$ and apoptosis inducing factor (AIF) in the cytoplasm, were measured using western blot and immunofluorescence labeling. To determine dissipation of mitochondrial membrane potential $(\Delta \Psi)$ though the permeability transition pore (PTP), the lipophilic cationic carbocyanine fluorescence probe $\mathrm{JC}-1$ and TMRM fluorescence probes were used. Results: Hypertonic culture conditions increase the abundance of proapoptotic Bax and the concentration of cytochrome $c$ and apoptosis inducing factor (AIF) in the cytoplasm. These changes are associated with a dissipation of $\Delta \Psi$ and increased permeability of the
\end{abstract}

\section{KARGER}

Fax +4161306 1234

E-Mail karger@karger.ch

www.karger.com
(C) 2010 S. Karger AG, Basel

$1015-8987 / 10 / 0256-0753 \$ 26.00 / 0$

Accessible online at:

www.karger.com/cpb
PTP. We further show that organic osmolytes stabilize the $\Delta \Psi$ and decrease the concentration of cytochrome $\mathrm{C}$ and AIF in the cytoplasm. Conclusion: Our study shows that organic osmolytes prevent hypertonicityinduced apoptosis by preventing dissipation of $\Delta \Psi$ through stabilization of the PTP. These findings further support the important role of organic osmolytes in preventing hypertonicity-mediated cell death in medullary kidney cells.

Copyright $(2010$ S. Karger AG, Basel

\section{Introduction}

Cells of the renal inner medulla are constantly exposed to a hypertonic environment that is incompatible with survival, unless cells accumulate organic osmolytes such as betaine, inositol, sorbitol and glycerophosphorylcholine (GPC) [1-5]. Intracellular concentrations of osmolytes are controlled by both rate of synthesis and transport. Myo-inositol and betaine transport is sodium-coupled, while sorbitol synthesis is catalyzed by the enzyme aldose reductase (AR). Both the sodium-myo-inositol (SMIT) [6] and betaine-GABA transporter 1 (BGT1) [7], as well as AR [8], are induced

Yale University School of Medicine, Department of Pathology

Renal Pathology and Electron Microscopy Laboratory

310 Cedar Street, LB20, PO Box 208023, New Haven, CT 06520-8023 (USA)

E-Mail Gilbert.Moeckel@yale.edu 
by hypertonicity in renal medullary cells in vitro and in vivo. Increased abundance of mRNA for SMIT, BGT1 and $\mathrm{AR}$ in response to hypertonicity depends on the binding of a transcription factor TonE-binding protein (TonEBP/ NFAT 5) [9] to a tonicity enhancer element (TonE) [10].

Under hypertonic conditions, ions such as $\mathrm{Na}^{+}, \mathrm{Cl}^{-}$ and $\mathrm{K}^{+}$, accumulate in the cytosol and are exchanged for compatible organic osmolytes that do not perturb intracellular protein structure or function $[5,3]$. Several studies have demonstrated that hypertonicity induces apoptosis in renal medullary interstitial cells (MICs) in vitro and in vivo [1,11]. One of these studies has shown that hypertonic culture conditions induce the effector caspase 3 , subsequently leading to apoptosis and that organic osmolytes significantly decrease apoptosis in MICs [1]. Furthermore, TonEBP/NFAT5 null mice show apoptotic cells in the renal medulla following dehydration, indicating that the absence of TonEBP/NFAT5, and therefore impaired osmolyte accumulation, decreases cell survival [12]. However, the cellular mechanisms by which organic osmolytes prevent activation of proapoptotic pathways are largely unknown.

Mitochondria play a pivotal role in cell death through the release of pro-apoptotic factors, which induce both caspase-dependent and caspase-independent cell death [13]. Increase in mitochondrial membrane permeability is the critical step for the release of proapoptotic proteins, such as cytochrome $\mathrm{c}$ and apoptosis inducing factor (AIF) from the intermembrane space. Mitochondrial permeability transition leads to mitochondrial membrane depolarization, an event that leads to cytochrome $\mathrm{c}$ release in apoptosis [14]. Mitochondrial permeability transition is widely believed to be caused by the opening of a regulated channel, the permeability transition pore (PTP). This channel, PTP, can be defined as a voltage-dependent, cyclosporine A(CsA) sensitive high conductance channel and its open-close transitions are highly regulated by multiple effectors [15]. The mechanisms by which Bcl-2 protein family members affect mitochondrial membrane potential have been intensely investigated [16]. Membrane potential can be regulated by targeting $\mathrm{Bcl} 2$ to the outer mitochondrial membrane and over expression of Bcl-2 prevents membrane depolarization [17]. Other proapoptotic Bcl-2 members, such as Bax, promote membrane depolarization and the release of proapoptotic factors such as cytochrome $\mathrm{c}$ and AIF $[18,19]$.

Hypertonicity-induced apoptosis is predominantly mediated through the intrinsic (mitochondrial) proapoptotic pathway [1]. Since the release of cytochrome $\mathrm{c}$ and AIF from mitochondria is an important step in the activation of this pathway, we examined whether osmolytes affect hypertonicity-induced rise in cytoplasmic cytochrome c and AIF. Furthermore, we explored the effect of osmolytes on hypertonicity-induced changes in Bcl-2 and Bax levels and on the dissipation of mitochondrial $\Delta \Psi$. We demonstrate that hypertonicity increases Bax, AIF and cytochrome $\mathrm{c}$ levels and dissipates the mitochondrial membrane potential. We show that osmolytes prevent dissipation of $\Delta \Psi$ by decreasing Bax and AIF levels and by stabilizing the PTP. Thus we provide evidence that the protective mechanisms by which organic osmolytes prevent apoptosis are twofold: first, preventing the dissipation of mitochondrial membrane potential through stabilization of the PTP leading to a decrease in AIF and second by decreasing Bax levels, thus preventing the release of cytochrome $\mathrm{c}$ from mitochondria.

\section{Materials and Methods}

\section{Cell culture}

Primary cultures of mouse medullary interstitial cells (MICs) were generated as described previously [1]. C57BL mice, obtained from The Jackson Laboratory (Bar Harbor, MA), were used for all experiments and were performed according to institutional animal care guidelines. Briefly, for the generation of primary cell cultures, both kidneys were rapidly removed under sterile conditions, inner medullary regions excised, and treated as previously described to generate MICs. Cells were then plated in 75-ml flasks and grown until confluent. Purity of the primary cell culture population was confirmed with oil red $\mathrm{O}$ stain under light microscopic examination as described previously [1]. MICs were studied after three or four passages and for experiments transferred to DMEM containing $2 \%$ fetal calf serum. The effect of hypertonicity on MICs was examined by increasing culture medium tonicity with $\mathrm{NaCl}$ to either 400 or $600 \mathrm{mosmol} / \mathrm{L}$. MICs were exposed to hypertonic medium for varying time periods, ranging from 5 minutes to 24 hours. In the experiments with betaine preincubation, MICs were incubated with culture medium of $350 \mathrm{mosmol} / \mathrm{L}$ containing 1 $\mathrm{mmol} / \mathrm{L}$ betaine prior to being exposed to higher tonicities.

\section{Immunofluorescent Studies}

MICs cultured on glass coverslips were plunged sequentially into methanol and acetone at $-20^{\circ} \mathrm{C}$, each for $10 \mathrm{~min}$. The cells were rehydrated in PBS, blocked with $1 \%$ bovine serum albumin in PBS for $15 \mathrm{~min}$, and stained with primary antibodies in blocking buffer for $1 \mathrm{~h}$ at room temperature. Primary antibodies were directed against apoptosis inducing factor (AIF, 1:100 dilution, Chemicon International, Temecula, CA), Bax (b-9, 1:200 dilution, Santa Cruz Biotechnology, Santa Cruz, CA) and Bcl-2 (1:200, Dako, Carpinteria, CA). Following incubation with primary antibody, cells were stained with either Texas Redconjugated goat anti-rabbit IgG (1:200 dilution), or fluorescein anti-mouse IgG $(\mathrm{H}+\mathrm{L})$ using a M.O.M. kit (Vector Laboratories 
Inc., Burlingame, CA). Cells were then rinsed with PBS and mounted in VectaShield mounting medium with DAPI (Vector Laboratories Inc.). For mitochondrial potential assessment, cells were loaded with tetramethylrodamine-methylester (TMRM) and calcein-ester fluorescence probes (Invitrogen, Carlsbad, CA). Cells were exposed to $1 \mu \mathrm{M} / \mathrm{L}$ Cyclosporine A (CsA) to examine the role of the PTP in hypertonicity-dependent dissipation of mitochondrial potential. Fluorescence was observed using a Zeiss AXIOVERT Imaging upright microscope driven by Metamorph software (Molecular Devices, Sunnyvale, $\mathrm{CA})$. Individual red and green stained images derived from the same field were merged using Openlab 3.1.5 software (Improvision Inc., Lexington, MA).

\section{Western Blot}

Following exposure to hypertonic medium, MICs were washed twice in PBS, lysed in ice cold RIPA buffer (Tris-HCL, $\mathrm{pH} 7.4,150 \mathrm{mM} \mathrm{NaCl}, 1 \% \mathrm{NP}-40,0.5 \%$ sodium deoxycholate, $0.1 \%$ SDS) with protease inhibitor cocktail (Hoffmann-La Roche Ltd., Basel, Switzerland) and incubated on ice for $30 \mathrm{~min}$. Protein content of the final extracts was estimated using the BCA kit according to the protocol of the manufacturer (Bio-Rad, Richmond, CA). Typically, $50 \mu \mathrm{g}$ protein was separated on SDSpolyacrylamide gel electrophoresis (PAGE) and electroblotted to nitrocellulose membranes. Biotinylated SDS-PAGE standards (broad range, Bio-Rad) were used for size determination. Proteins were detected by the ECL Western blotting analysis system (Amersham Pharmacia, Arlington Heights, IL). The following primary antibodies were used: purified anticytochrome C, anti-Fas (CD95) and anti-Fas ligand (CD178) (Biosciences Pharmingen, San Jose, CA); anti-Bcl-2 and antiBax antibodies (Santa Cruz Biotechnology, Santa Cruz, CA), cleaved anti-caspase- 8 and caspase-9 antibodies (Cell Signaling, Beverly, MA). Secondary antisera were anti-rabbit IgG Horseradish Peroxidase linked whole antibody and antimouse IgG Horseradish Peroxidase linked whole antibody (Amersham Biosciences). Membranes were exposed to Kodak Scientific Imaging film. To determine densities of protein bands blots were analyzed using $\alpha$-imaging software (Bio-Rad).

\section{Isolation of Mitochondria}

Mitochondrial and cytosolic fractions of MICs were isolated as described previously [20]. MICs were centrifuged at $600 \mathrm{~g}$ for 10 minutes, cell pellets were washed once with icecold PBS, spun down and resuspended with five volumes of buffer A (0.25 M sucrose, $20 \mathrm{mM}$ HEPES-KOH pH 7.4, $10 \mathrm{mM}$ $\mathrm{KCl}, 1.5 \mathrm{mM} \mathrm{MgCl}, 1 \mathrm{mM}$ EDTA and $1 \mathrm{mM}$ sodium EGTA, $1 \mathrm{mM}$ dithiothreitol, $1 \mathrm{mM}$ PMSF) and homogenized with 10 strokes using a teflon homogenizer. Homogenates were centrifuged twice at $750 \mathrm{~g}$ for $10 \mathrm{~min}$ at $4{ }^{\circ} \mathrm{C}$. Supernatants were then centrifuged at $10,000 \times \mathrm{g}$ for 10 minutes at $4{ }^{\circ} \mathrm{C}$, the pellets resuspended in buffer A and stored at $-80{ }^{\circ} \mathrm{C}$. Supernatants of the previous $10,000 \mathrm{x} \mathrm{g}$ spin were then further centrifuged at $100,000 \mathrm{x}$ g for 1 hour at $4{ }^{\circ} \mathrm{C}$. Supernatants from this centrifugation were also stored at $-80{ }^{\circ} \mathrm{C}$ for further experiments. In both mitochondrial and cytosolic fractions, total amounts of lactate dehydrogenase (cytosolic marker) and glutamate dehydrogenase (mitochondrial marker) were measured. Protein

Hypertonicity-induced Mitochondrial Membrane Permeability

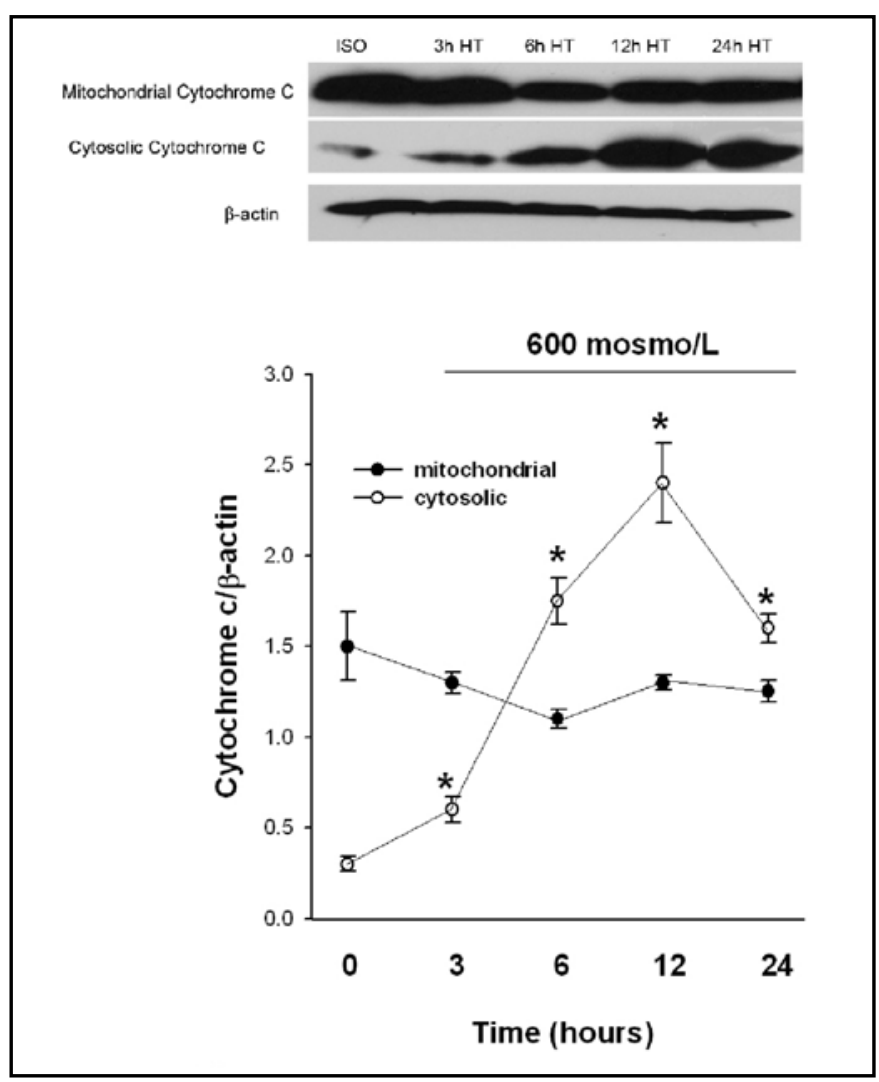

Fig. 1. Cytochrome c levels in cytosolic and mitochondrial fractions of medullary interstitial cells (MICs) following exposure to $600 \mathrm{mosmol} / \mathrm{L}$ for 3, 6, 12 and 24 hours. Cytosolic cytochrome $\mathrm{c}$ abundance was markedly increased at 6 and 12 hours. Cytochrome c levels were determined using western blot; iso=isotonic medium (295 mosol/L), h= hours, $\mathrm{HT}=$ hypertonic culture medium. All values are mean $\pm \mathrm{SEM}$ of $\mathrm{n}=4$. ${ }^{*} \mathrm{p}<0.05$ compared to isotonic control at 0 hours.

concentrations were determined using the method described by Bradford [21]. Relative specific ativities (RSA) of LDH and GDH were calculated as specific activities ${ }_{\text {fraction }} /$ specific activities homogenate. $\mathrm{RSA}_{\mathrm{GDH}}$ in mitochondrial fractions was 2.20 compared to 0.15 in the cytosolic fraction. $\mathrm{RSA}_{\mathrm{LDH}}$ in the mitochondrial fraction was 0.45 compared to 4.71 in the cytosolic fraction.

\section{Measurement of $\Delta \psi$ in Isolated Mitochondria}

To determine $\Delta \psi$ in isolated mitochondria to assess the integrity of mitochondrial membrane following exposure to isotonic and hypertonic buffer conditions, the lipophilic cationic carbocyanine fluorescence probe JC-1 was used.

To monitor the kinetics of changes in $\Delta \psi$ during hypertonic stress treated with or without osmolytes, mitochondria $(1 \mathrm{mg} / \mathrm{ml})$ were incubated with $\mathrm{JC}-1(5 \mathrm{ug} / \mathrm{ml})$ in PBS at different time points and fluorescence intensity was recorded at room temperature using fluorescence microplate reader (Fluoroskan Ascent FL, Labsystens OY, Helsinki, Finland). An excitation wavelength of $485 \mathrm{~nm}$ was used, and emission by the dye monomers and aggregates was measured at $538 \mathrm{~nm}$ (green) and $590 \mathrm{~nm}$ (orange-red), respectively. A 


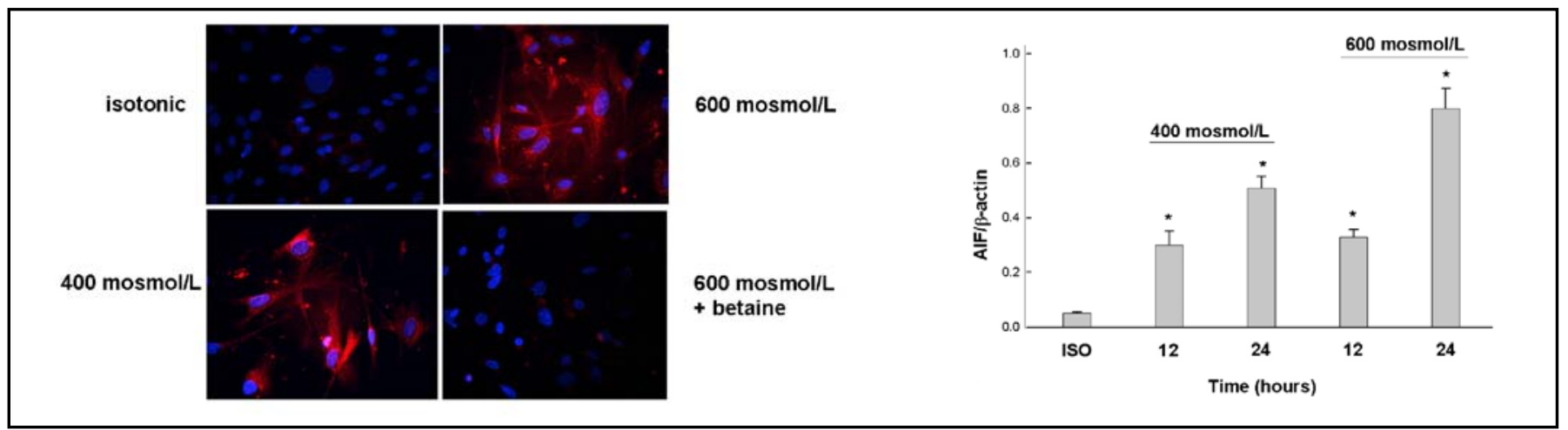

Fig. 2. Immunofluorescence labeling (Texas red) of Apoptosis Inducing Factor (AIF) in medullary interstitial cells (MICs) under isotonic and hypertonic conditions (400 and 600 mosmol/L for 12 hours). Preincubation of MICs with 1 mmol/L betaine followed by exposure to $600 \mathrm{mosmol} / \mathrm{L}$ for 12 hours markedly decreased AIF staining (right lower quadrant). AIF/ $\beta$-actin ratio was determined by western blot in MICs exposed to 400 and $600 \mathrm{mosmol} / \mathrm{L}$ for 12 and 24 hours respectively. All values are mean \pm SEM of $\mathrm{n}=4 ; * \mathrm{p}<0.05$ compared to isotonic controls.

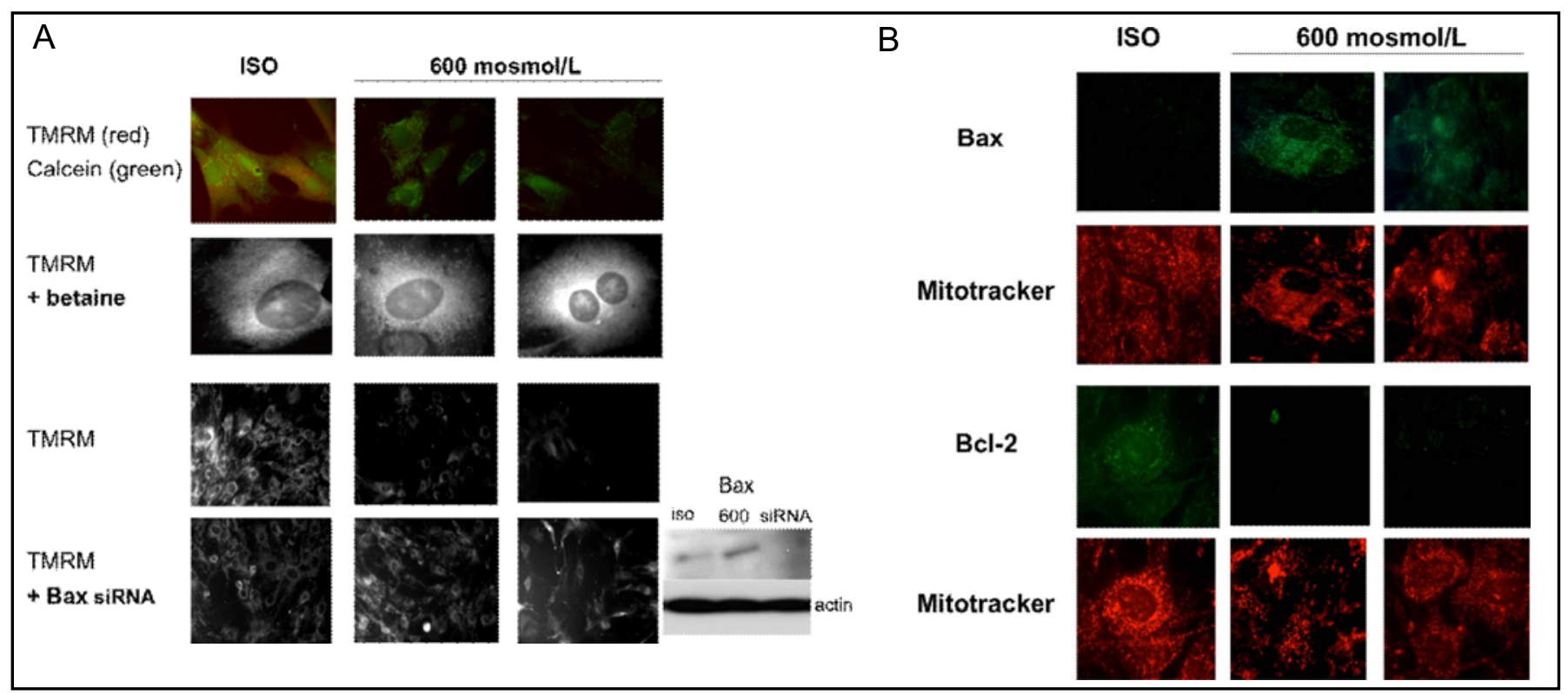

Fig. 3. A: Medullary interstitial cell (MIC) mitochondria were labeled with TMRM ( $5 \mu \mathrm{M}$, red) and calcein $\mathrm{AM}(1 \mu \mathrm{M}$, green) under isotonic conditions, followed by exposure to 600 mosmol/L medium tonicity for 6 hours (middle column) and 12 hours (right column). Hypertonicity induced rapid loss of TMRM and calcein fluorescence over the course of 12 hours (top row, right image). Preincubation of MICs with $1 \mathrm{mM} / \mathrm{L}$ betaine preserved mitochondrial potential as shown by persistent strong TMRM stain over 12 hours. Knock down of Bax (insert) using silencing RNA also increased TMRM staining over 12 hours of hypertonic exposure (bottom row, right image). TMRM= tetramethylrhodamine methyl ester, iso=isotonic conditions. B: Cytoplasmic Bax levels increased in MICs that were exposed to $600 \mathrm{mosmol} / \mathrm{L}$ for 6 (middle column) and 12 hours (right column). After 12 hours of hypertonic medium, Bax fluorescence (green, top row, right image) was strongest in mitochondria (co-labeled with MitoTracker, red, second row from top, right image). To the contrary Bcl-2 fluorescence (green, second row from bottom) was markedly decreased at 6 and 12 hours of exposure to $600 \mathrm{mosmol} / \mathrm{L}$ (middle and right image). Bcl-2 primarily located in mitochondrial regions of the cytoplasm as shown by the Mitotracker stained organelles (red, bottom row) under isotonic conditions. Exposure to $600 \mathrm{mosmol} / \mathrm{L}$ medium showed persistent mitotracker staining of mitochondria for 6 and 12 hours (bottom row). Images are representative of four experiments.

ratiometric method was used with this dye $(590 / 538 \mathrm{~nm})$ to provide a semi-quantitative measurement of $\Delta \psi$. Carbonyl cyanide 3 - chlorophenylhydrazone (CCCP, 50uM), was added to mitochondrial suspensions as a positive control to verify the sensitivity of JC-1 to $\Delta \psi$.

\section{Statistics}

Results are presented as mean \pm S.D. of 4-6 replicates. Differences were considered statistically significant if $\mathrm{p}<0.05$, using analysis of variance and Bonferroni t tests. 


\section{Results}

Organic osmolytes prevent cytochrome $c$ and AIF release to cytoplasm

Release of cytochrome $\mathrm{c}$ and Apoptosis inducing factor (AIF) from the mitochondrial intermembrane space is an initiating step in the induction of the mitochondrial proapoptotic pathways [22-24]. To examine the effect of hypertonic stress on mitochondrial cytochrome c release, we exposed MICs to $600 \mathrm{mosm} / \mathrm{L}$ for 3, 6, 12 and 24 hours followed by measurement of cytochrome $\mathrm{c}$ in mitochondrial and cytoplasmic fractions. Three hours of exposure to hypertonic medium initiated cytochrome $\mathrm{c}$ release from mitochondria. Cytoplasmic cytochrome c levels continued to increase over the following 9 hours and peaked 12 hours after onset of hypertonicity (Fig. 1). Moreover, MICs exposed to medium tonicity of 400 and $600 \mathrm{mosm} / \mathrm{L}$ for 12 and 24 hours showed significant increases in cytoplasmic AIF levels, both by western blot and Texas-red immunofluorescence. Preloading of MICs with $1 \mathrm{mmol} / \mathrm{L}$ betaine significantly decreased AIF concentration in the cytosol (Fig. 2).

Hypertonicity affects the mitochondrial permeability transition pore

The mitochondrial permeability transition pore (PTP) is an important regulator of the mitochondrial membrane potential. The PTP plays an important role in the release of proapoptotic factors such as cytochrome $\mathrm{c}$ and AIF from the mitochondrial intermembrane space [25]. We examined the effect of hypertonicity on the PTP and the mitochondrial membrane potential $(\Delta \Psi)$. Cells were loaded with the membrane potential-sensitive dye TMRM and esterified calcein, followed by exposure to hypertonic medium for 6 and 12 hours. Under isotonic conditions mitochondria accumulated both TMRM (red fluorescence) and calcein (green fluorescence, Fig. 3A first row, left image). Increase in medium tonicity to 600 mosmol/L decreased fluorescence of both dyes (Fig. 3A first row, middle and right image). Incubating cells with 1 $\mathrm{mmol} / \mathrm{L}$ betaine at $350 \mathrm{mosmol} / \mathrm{L}$, prior to elevating tonicity to $600 \mathrm{mosmol} / \mathrm{L}$, preserved mitochondrial TMRM fluorescence (Fig. 3A, second row, middle and right image). Bax knock down with Bax siRNA also prevented hypertonicity-induced dissipation of TMRM fluorescence (Fig. 3A, last row).

Hypertonicity affects Bcl-2 and Bax levels

We further examined the effect of hypertonicity on the pro- and anti-apoptotic Bcl-2 family members Bax

Hypertonicity-induced Mitochondrial Membrane Permeability

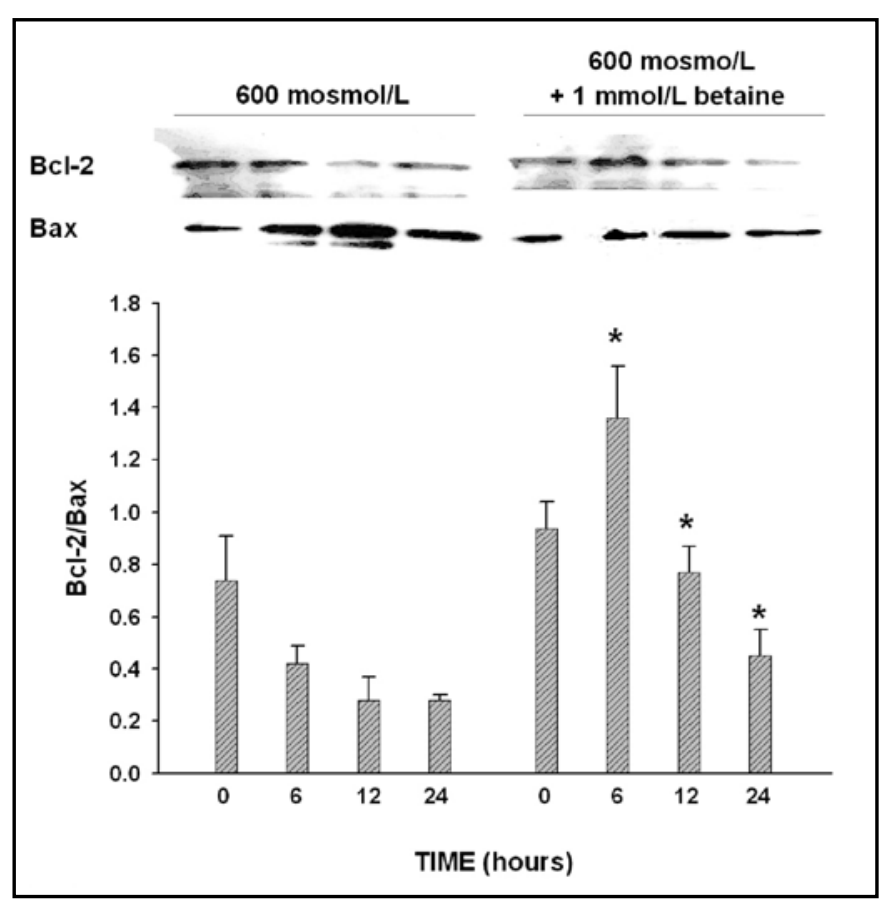

Fig. 4. Bcl-2/Bax ratio was determined by western blot in MICs under isotonic conditions ( 0 hour) and after exposure to 600 mosmol/L medium tonicity for 6,12 and 24 hours. Compared to isotonic controls, the $\mathrm{Bcl}-2 / \mathrm{Bax}$ ratio markedly decreased at 6 , 12 and 24 hours following exposure to hypertonic culture conditions. Preincubation of MICs with $1 \mathrm{mmol} / \mathrm{L}$ betaine markedly inhibited Bax expression at 6,12 and 24 hours of hypertonicity and reversed the ratio in favor of Bcl-2. Western blot is representative of four individual experiments. Values in graph blot are mean \pm SEM of $n=4 .{ }^{*} p<0.05$ compared to identical time point without betaine treatment.

and Bcl-2. Bax levels significantly increased following exposure to $600 \mathrm{mosmol} / \mathrm{L}$ medium for 6 and 12 hours (Fig. 3B, first row, middle and right image). Bax was predominantly located close to the mitochondria, which could be visualized using red fluorescent Mitotracker dye (Fig. 3B, second row from top, middle and right image). Exposure to $600 \mathrm{mosmol} / \mathrm{L}$ medium tonicity decreased mitochondrial BcL-2 levels (Fig. 3B, third row, middle and right image) which was predominantly localized to the mirochondria under sotonic conditions (Fig. 3B, bottom row, left image). As shown by western blot in Fig. 4, the Bcl-2/Bax ratio decreased in MICs grown at $600 \mathrm{mosmol} /$ $\mathrm{L}$ and was reversed by preloading cells with betaine at $400 \mathrm{mosmol} / \mathrm{L}$, prior to increasing the medium tonicity to $600 \mathrm{mosmo} / \mathrm{L}$.

Hypertonicity affects Mitochondrial Membrane Permeability

Mitochondrial membrane potential was measured in isolated mitochondria under isotonic and hypertonic 
Fig. 5. Isolated mitochondria $(1 \mathrm{mg} / \mathrm{ml})$ from medullary interstitial cells were suspended in isotonic and hypertonic (600 mosmol/L) PBS buffer for 30 or 60 minutes and changes in mitochondrial membrane potential $(\Delta \psi)$ were measured by the JC-1 fluorescence ratio of red to green (see methods). CCCP $(50 \mu \mathrm{M})$ was used as positive control. The osmolytes betaine, inositol or sorbitol were added to hypertonic buffer $(600 \mathrm{msmol} /$ $\mathrm{L}, \mathrm{PBS}$ ) at $1 \mathrm{mmol} / \mathrm{L}$. Hypertonicity dissipated $\Delta \Psi$ to a similar degree as CCCP. Addition of betaine, inositol and sorbitol significantly diminished $\Delta \psi$ dissipation. ${ }^{*} \mathrm{p}<0.05$ compared to samples at identical time points and $600 \mathrm{mosmol} / \mathrm{L}$ medium tonicity without osmolyte supplementation.

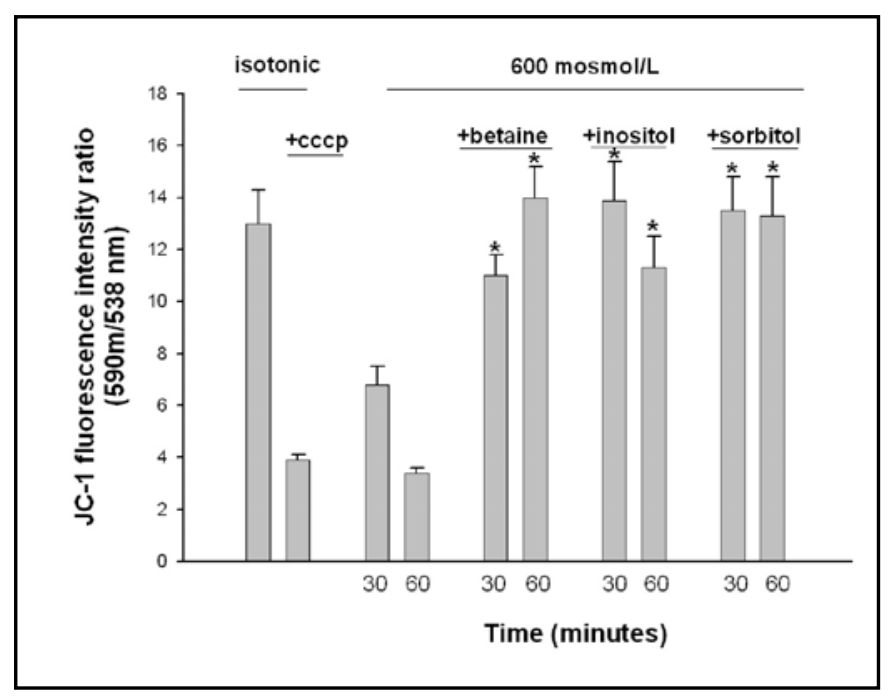

Fig. 6. Medullary interstitial cells (MICs) were loaded with TMRM and exposed to $600 \mathrm{mosmol} / \mathrm{L}$ for 12 hours with or without Cyclosporine A (CsA). CCCP $(50 \mu \mathrm{M})$ was used as positive control to dissipate the mitochondrial membrane potential both under isotonic and hypertonic conditions (top and middle row, middle images). Hypertonic medium caused significant loss of membrane potential, which was prevented by CsA (middle row, left and right images). Preloading MICs with organic osmolytes $(1 \mathrm{mmol} / \mathrm{L}$ medium concentration), prior to exposure to 600 mosmol/L for 12 hours, preserved mitochondrial as shown by persistent TMRM fluorescence.

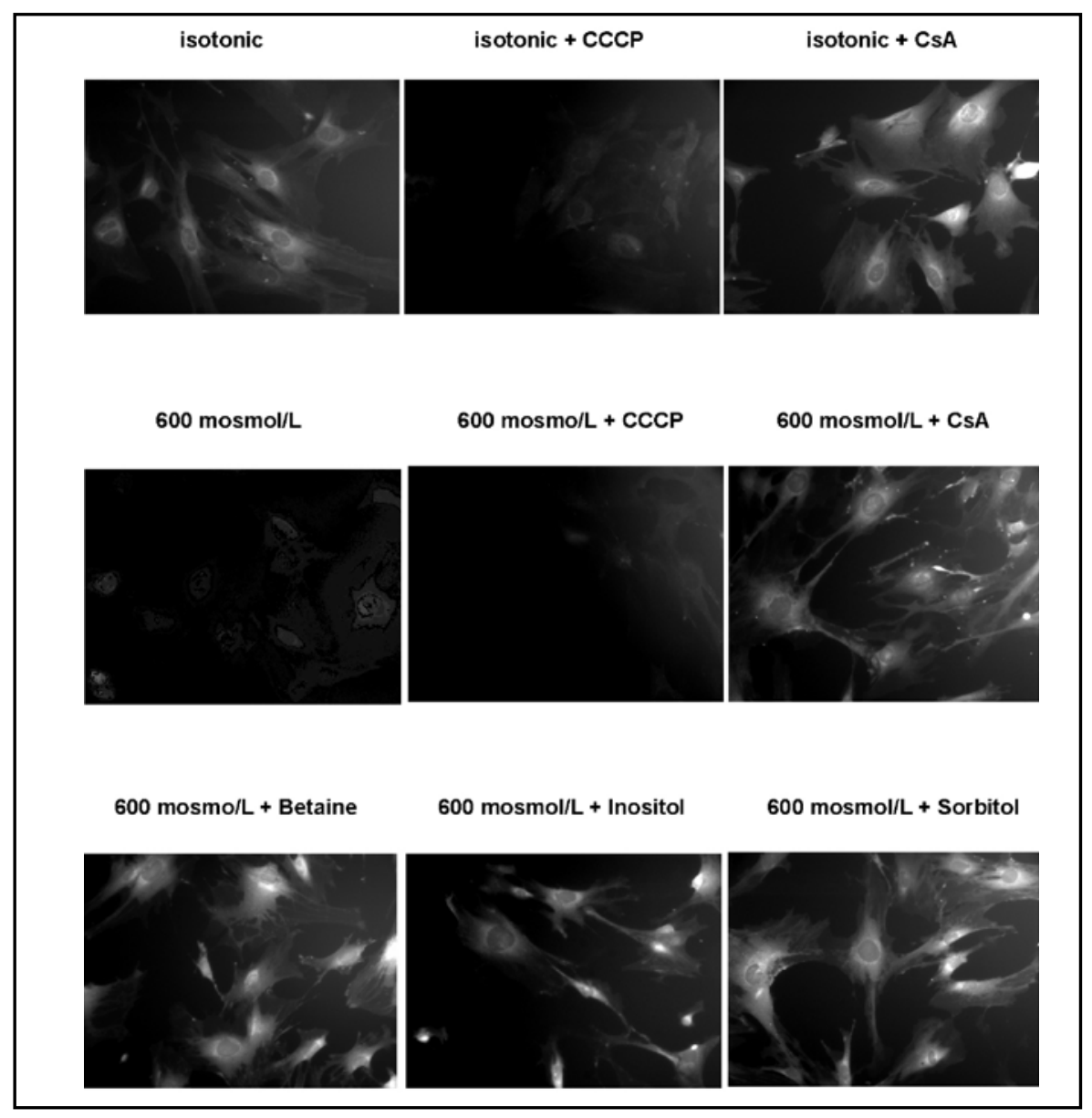

conditions, with and without addition of individual osmolytes, using the JC-1 fluorescence ratio of red to green (Fig. 5). Carbonyl cyanide 3-chlorophenylhydrazone (CCCP), a depolarizing agent of the mitochondrium, induced dissipation of the mitochondrial potential and lowered the $\Delta \Psi$ by $70 \%$. Exposure of isolated mitochondria to hypertonic buffer $(600 \mathrm{mosmol} / \mathrm{L})$ for 60 minutes showed a similar degree of $\Delta \Psi$ dissipation. Addition of betaine, inositol or sorbitol to the hypertonic buffer prevented the dissipation of $\Delta \Psi$.

To further examine the role of the PTP in hypertonicity-induced dissipation of the mitochondrial membrane potential, we exposed medullary interstitial cells to hypertonic medium with or without CCCP and Cyclosporine A(CsA), a stabilizer of the PTP. Reduction in TMRM staining following exposure to both hypertonicity and CCCP is shown in Fig. 6 (upper and middle row). CsA prevented the dissipation 
of mitochondrial membrane potential under hypertonic conditions (Fig. 6, middle row, right image). Organic osmolytes betaine, inositol and sorbitol prevented dissipation of mitochondrial potential under hypertonic culture conditions (Fig 6, bottom row).

\section{Discussion}

In a previous study we showed that hypertonic culture medium induces apoptosis in MICs and that organic osmolytes prevent this [1]. This study is aimed at investigating the mechanisms how osmolytes prevent hypertonicity-induced apoptosis in MICs. We show that hypertonic culture conditions markedly stimulate mitochondrial depolarization. Hypertonic medium induces Bax expression and Bax siRNA under these conditions prevents dissipation of the mitochondrial potential. Hypertonic culture conditions further increased cytochrome c and AIF levels in the cytosol. We report the novel finding that osmolytes prevent the hypertonicityinduced depolarization of mitochondria and decrease the subsequent release of cytochrome $\mathrm{c}$ and AIF to the cytosol. We speculate that this protective effect of organic osmolytes is mediated by decreasing the mitochondrial membrane permeability. Possible mechanisms that could explain how osmolytes mediate these effects include inhibiting Bax or directly stabilizing the PTP.

MICs exposed to hypertonic culture medium for several hours showed dissipation of mitochondrial potential and subsequent release of proapoptotic factors cytochrome $\mathrm{c}$ and apoptosis inducing factor (AIF). Previous studies in both isolated mitochondria and intact cells, using damaging agents other than hypertonic buffer, have shown that cytochrome c release takes place following dissipation of mitochondrial potential $[25,26]$. Moreover, high $\mathrm{NaCl}$ conditions have been shown to induce cell cycle delay and mitochondrial injury in kidney cells from the medulla [27, 28]. Our findings in isolated mitochondria from MICs indicate that hypertonicity dissipates the mitochondrial potential $(\Delta \psi)$ and that CsA, a stabilizer of the permeability transition pore (PTP), and osmolytes prevent this dissipation. The protective effect of osmolytes might be exerted in a similar fashion as the protective effect of CSA, by stabilizing the PTP. Supporting this conclusion is the observation that trehalose improves inner mitochondrial membrane integrity and decreases mitochondrial potential loss secondary to

Hypertonicity-induced Mitochondrial Membrane Permeability desiccation [29]. A recent study showed that release of cytochrome $\mathrm{c}$ from mitochondria is dependent upon electrostatic interactions between cytochrome $\mathrm{c}$ and mitochondrial membranes [30]. Osmolytes might have a modulating effect on electrostatic association of cytochrome $\mathrm{c}$ to mitochondrial membranes, which might further play a role in preventing cytochrome $\mathrm{c}$ release to the cytosol.

Another possible mechanism how osmolytes might prevent apoptosis is by inhibiting the action of Bax. Hypertonicity-induced stimulation of Bax might be an important step that induces leakage of cytochrome $\mathrm{c}$ and AIF to the cytosol. Activated Bax, which has intrinsic pore-forming activity, may permeabilize the mitochondrial outer membrane and induce leakage of proapoptotic factors to the cytosol [31]. Translocation of Bax from the cytoplasm to the mitochondrial outer membrane is a regulatory step prior to cytochrome c release in other cell models of apoptosis [32]. Bax forms selective channels for cytochrome $\mathrm{c}$ and AIF release from the intermembrane space $[33,34]$. Our study shows that Bax might play a role in hypertonicity-induced dissipation of mitochondrial potential. This could be mediated through Bax-dependent pore formation or Bax-induced destabilization of the PTP. Preloading cells with betaine prior to hypertonic stress markedly reduced Bax expression and subsequent AIF release. Organic osmolytes might have a negative effect on Bax-mediated pore formation or might prevent the destabilizing effect of Bax on the PTP. Further studies are needed to examine these possibilities.

In summary we report an important novel observation that organic osmolytes prevent mitochondrial depolarization and the efflux of the mitochondrial proapoptotic factors cytochrome $\mathrm{c}$ and AIF in hyperosmotically stressed medullary interstitial cells. Osmolytes decrease mitochondrial membrane permeability through yet unknown action on the mitochondrial PTP or by reducing Bax-mediated pore formation. Our study indicates that organic osmolytes are important for the prevention of apoptosis in medullary interstitial kidney cells.

\section{Acknowledgements}

This work was supported by National Institutes of Health Grants KO8 DK59975 and RO3 DK 07770 (to GWM). 


\section{References}

$>1$ Moeckel GW, Zhang L, Fogo AB, Hao CM, Pozzi A, Breyer MD: COX2 activity promotes organic osmolyte accumulation and adaptation of renal medullary interstitial cells to hypertonic stress. J Biol Chem 2003;278:1935219357.

-2 Yancey PH, Clark ME, Hand SC, Bowlus RD, Somero GN: Living with water stress: Evolution of osmolyte system. Science 1982;217:1214-1222.

3 Garcia-Perez A, Burg MB: Renal medullary organic osmolytes. Physiol Rev 1991;71:1081-1115.

$\checkmark 4$ Kwon ED, Zablocki K, Jung KY, Peters EM, Garcia-Perez A, Burg MB: Osmoregulation of GPC:choline phosphaodiesterase in MDCK cells: different effects of urea and $\mathrm{NaCl}$. Am J Physiol 1995;269:C35-C41.

$\checkmark 5$ Neuhofer W, Beck FX: Cell Survival in the hostile environment of the renal medulla. Annu Rev Physiol 2005;67:53155.

-6 Yamauchi AS, Uchida AS, Preston AS, Kwon HM, Handler JS: Hypertonicity stimulates transcription of gene for $\mathrm{Na}+$ myo-inositol cotransporter in MDCK cells. Am J Physiol 1993;264:F20-F23.

7 Uchida SA, Yamauchi AS, Preston AS, Kwon MH, Handler JS: Medium tonicity regulates expression of the $\mathrm{Na}+$ and $\mathrm{Cl}$ dependent betaine transporter in MadinDarby canine kidney cells by increasing transcription of the transporter gene. J $>20$ Clin Invest 1993;91:1604-1607.

$>8$ Smardo FL, Burg MB Garcia-Perez A: Kidney aldose reductase gene transcription is osmotically regulated. Am J Physiol 1992;262:C776-C782.

$\checkmark 9$ Miyakawa H, Woo SK, Dahl SC, Handler $>21$ JS, Kwon HM: Tonicity-responsive enhancer binding protein, a rel-like protein that stimulates transcription in response to hypertonicity. Proc Natl Acad Sci 1999;96:2538-2542.

10 Takenaka M, Preston AS, Kwon HM, Handler JS: The tonicity-sensitive element that mediates increased transcription of the betaine transporter gene in response to hypertonic stress. J Biol Chem 1994;269:29379-29381.

11 Hao C, Yull F, Blackwell T, Komhoff M, Davis L, Breyer MD: Dehydration activates an NF-kappaB-driven, COX2dependent survival mechanism in renal medullary interstitial cells J Clin Invest 2000;106:973-982.

$>12$ Lopez-Rodriguez C, Antos CL, Shelton JM, Richardson JA, Lin F, Novobrantseva TI, Bronson RT, Igarashi P, Rao A, Olson EN: Loss of NFAT5 results in renal atrophy and lack of tonicity-responsive gene expression. Proc Natl Acad Sci 2004;101:2392-2397.
Green DR, Kroemer G: Pharmacological 25 manipulation of cell death: clinical applications in sight? J Clin Invest 2005;115:2610-2617.

14 Garlid KD. On the mechanism of regulation of the mitochondrial $\mathrm{K}+/ \mathrm{H}+$ exchanger. J Biol Chem 1980;255:1127311279 .

15 Massari S, Azzone GF: The equivalent pore radius of intact and damaged mitochondria and the mechanism of active shrinkage. Biochim. Biophys Acta 1972;283:23-29.

16 Green DR, Kroemer G: Pathophysiology of mitochondrial cell death. Science 2004;305:626-629.

17 Susin SA, Zamzami N, Castedo M, Hirsch T, Marchetti P, Macho A, Dougas E, Geuskens M, Kroemer G: Bcl-2 inhibits the mitochondrial release of an apoptogenic protease. J Exp Med 1996;184:1331-1341.

18 Gross A, Korsmeyer SJ: Bcl2 family members and the mitochondria in apoptosis. Genes Dev 1999;13:18991911.

19 Choi SY, Kim MJ, Kang CM, Bae S, Cho CK, Soh JW, Kim JH, Kang S, Chung HY, Lee YS Lee SJ: Activation of Bak and Bax through c-abl-protein kinase Cdeltap38 MAPK signaling in response to ionizing radiation in human non-small cell lung cancer cells. J Biol Chem 2006;281:7049-7059.

0 Yang J, Liu X, Bhalla K, Kim CN, Ibrado AM, Cai J, Peng TI, Jones DP, Wang X: Prevention of apoptosis by BCL-2: Release of cytochrome $\mathrm{c}$ from mitochondria blocked. Science 1997;275:1129-1132.

Bradford MM. A rapid and sensitive method for the quantification of microgram quantities of protein utilizing the principle of protein-dye binding. Anal Biochem 1976;72:248-254.

-22 Ott M, Robertson JD, Gogvadze V, Zhivotovsky B, Orrenius S. Cytochrome $\mathrm{c}$ release from mitochondria proceeds by a two-step process. Proc Natl Acad Sci 2002;99:1259-1263.

23 Horio M, Ito A, Matsuoka Y, Miriyama T, Orita Y, Takenaka M, Imai E: Apoptosis induced by hypertonicity in Madin-Darbey canine kidney cells: protective effect of betaine. Nephrol Dial Transplant 2001;16:483-490.

24 Lorenzo HK, Santos AS, Penninger J, Kroemer G: Apoptosis inducing factor 34 (AIF): a phylogenetically old, caspaseindependent effector of cell death. Cell Death Differ 1999;6:516-524.
Kluck RM, Bossy-Wetzel E, Green DR, Newmeyer DD: The release of cytochrome c from mitochondria: a primary site for Bcl-2 regulation of apoptosis. Science 1997;275:1132-1136. Kluck RM, Esposti MD, Perkins G, Renken C, Kuwana T, Bossy-Wetzel E, Goldberg M, Allen T, Barber MJ, Green DR, Newmeyer DD: The pro-apoptotic proteins Bid and Bax cause a limited permeabilization of the mitochondrial outer membrane that is enhanced by cytosol. J Cell Biol 1999;147:809-822. Michea L, Ferguson DR, Peters EM, Andrews PM, Kirby MR, Burg MB: Cell cycle delay and apoptosis are induced by high salt and urea in renal medullary cells. Am J Physiol Renal Physiol 2000;278:F209-F218.

Michea L, Combs C, Andrews P, Dmitrieva N, Burg MB: Mitochondrial dysfunction is an early event in highNaCl-induced apoptosis of mIMCD3 cells. Am J Physiol Renal Physiol 2002;282:F981-F990.

29 Liu HX, Aksan A, Menze M, Hand S, Toner M: Trehalose loading through the mitochondrial permeability transition pore enhances desiccation tolerance in rat liver mitochondria. Biochim Biophys Acta 2005;1717:21-26.

Uren RT, Dewson G, Bonzon C, Lithgow T, Newmeyer DD, Kluck RM: Mitochondrial release of proapoptotic proteins. J Biol Chem 2005;280:22662274.

Kuwana T, Mackey M, Perkins G, Ellisman MH, Latterich M, Schneiter R, Green D, Newmeyer DD: Bid, Bax, and lipids cooperate to form supramolecular openings in the outer mitochondrial membrane. Cell 2002;111:331-342.

Saikumar P, Dong Z, Patel Y, Hall K, Hopfer U, Weinberg JM, Venkatachalam MA: Role of hypoxia-induced Bax translocation and cytochrome c release in reoxygenation injury. Oncogene 1998;17:3401-3415.

Susin SA, Lorenzo HK, Zamzami N, Marzo I, Snow BE, Brothers GM, Mangion J, Jacotot E, Costantini P, Loeffler M, Larochette N, Goodlett DR, Aebersold R, Siderowski DP, Penninger JM, Kroemer G: Molecular characterization of mitochondrial apoptosis-inducing factor. Nature 1999;397:441-446.

Vander Heiden MG, Chandel NS, Williamson EK, Schumacker PT, Thompson CB: Bcl-xl regulates the membrane potential and volume homeostasis of mitochondria. Cell 1997;91:627-637. 